\title{
FREE VIBRATION OF VARIABLE THICKNESS PLATES USING CHARACTERISTIC ORTHOGONAL POLYNOMIAL STRIP FUNCTIONS SUBJECTED TO DIFFERENT COMBINATIONS OF BOUNDARY CONDITIONS
}

\author{
A. A. Rizk` and A. S. Ashour* ${ }^{*}$ \\ Department of Science in Engineering, Faculty of Engineering, International Islamic University Malaysia, \\ 53100 Kuala Lumpur, Malaysia \\ Email:, ashour@iiu.edu.my
}

\begin{abstract}
A set of characteristic orthogonal polynomial strip functions was used to study the flexural vibration of variable thickness plates in one direction. The starting function in the set, which is satisfying the geometry and natural conditions, is developed from the solution of a static beam subjected to an arbitrary load that expanded into a Taylor series. The Gram-Schmidt orthogonalization process is used to generate the reminder functions of the set. The natural frequencies of the plat are calculated by using the finite strip method together with the transition matrix. Numerical results are obtained for different combination of boundary conditions, tapered ratio and aspect ratio. It is demonstrated that the method as well as the orthogonal strip functions yield good accuracy and fast convergence by comparisons with available results in the literature. This technique can also be used to solve some other complicated problems in the engineering applications.
\end{abstract}

\section{INTRODUCTION:}

Plates of variables thickness are commonly used as structural elements in many engineering applications such as aerospace, civil, and ocean engineering systems. The natural frequencies and mode shapes of the plate are useful in their operation environments. In the literature, there are many publications concerning the flexural vibration of uniform and non-uniform plates, see for examples Lissisa ${ }^{[1-5]}$. In last decades, many of the researchers are used different admissible functions to obtain the natural frequencies of rectangular and skew plates of variable thickness. Ashour ${ }^{[6]}$ used the eigen functions derived from the solution of a classical beam vibration as basis functions. Cheung and Zhou ${ }^{[7]}$ used a set of admissible functions that are developed from static solution of beam under arbitrary Taylor series load in Rayleigh-Ritz method. Zhou ${ }^{[8]}$ used a combination of sine series and polynomials as the basis functions in Rayleigh-Ritz method. Grossi and Bhat ${ }^{[9]}$ used boundary characteristic orthogonal polynomials in Rayleigh-Ritz method and applied Rayleigh-Schmidt method in their analysis. Singh and Saxena ${ }^{[10-12]}$ used boundary characteristic orthogonal polynomials in two variables in Rayleigh-Ritz method. Also Lewi and lam ${ }^{[13]}$, and Bhat ${ }^{[14]}$ used two-dimensional orthogonal polynomials. Malhotra ${ }^{[15]}$ used the conventional beam functions as the basis functions. Bhat ${ }^{[16]}$ used a set of orthogonal polynomial functions in Rayleigh-Ritz method.

In this paper, a set of orthogonal polynomial shape functions in $\mathrm{x}$-direction is developed to study the free vibration of variable thickness plate in y-direction. The first strip function in the set that satisfies the geometry and natural boundary conditions is the first term in the series solution obtained by Cheung and Zhou ${ }^{[7]}$ as a new set of admissible functions that are the static solution of the beam under an arbitrary static load expanded into Taylor series. The rest of the orthogonal polynomial strip functions are generated through GramSchmidt orthogonalization process, which are also satisfy the geometry and natural boundary conditions. It is shown that, this set is the same as developed by Bhat [16]. The finite strip method in conjunction with transition matrix ${ }^{[17-18]}$ is used to calculate the natural frequencies of the plate for different boundary conditions, tapered ratio and aspect ratio. Good accuracy and fast convergence for all cases presented in this paper are demonstrated when compared with available results in the literature.

\section{THE CHARACTERISTIC ORTHOGONAL POLYNOMIAL STRIP FUNCTIONS:}

One of the admissible functions that are generated by Cheung and Zhou ${ }^{[7]}$ is adopted in the present work. Consider a beam of length equal $l$ and unit width under an arbitrary static load $p(x)$. If the width and the depth of the beam are small compared to its length, the classical beam theory is valid. The non-dimensional

'On leave from Faculty of Engineering, Alexandria University, Alexandria, Egypt.

${ }^{\star}$ Corresponding Author 
differential equation that is governing the static deflection of the beam may be put in the form

$\frac{\mathrm{d}^{4} y}{\mathrm{~d} x^{4}}=P(x)$

where $x=\frac{x}{l}, P(x)=\frac{l^{4}}{E I} p(x)$ and $E I$ is the flexural rigidity of the beam. The arbitrary static load $P(x)$ can be expanded into Taylor series as

$P(x)=\stackrel{\stackrel{\ddagger}{\circ}}{\stackrel{\mathrm{a}}{\mathrm{a}}} P_{i}^{\prime} x^{i}$

where $P_{i}^{\prime}$ are the constants that can be determined if $P(x)$ is a given function. Using equation (2) into equation (1), the general solution of the differential equation can be put in the form

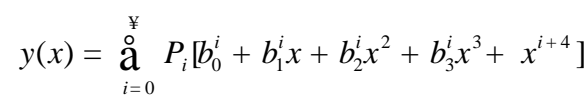

The first term in the series solution will be taken as the first strip function in the set of orthogonal polynomial strip functions, i.e.

$y_{o}(x)=a_{o}+a_{1} x+a_{2} x^{2}+a_{3} x^{3}+x^{4}$

Where $a_{i}(i=0,1,2,3)$ are arbitrary constants to be determined from the boundary conditions. For clamped ends

$y_{o}(0)=y_{o}(1)=\frac{d y_{o}(0)}{d x}=\frac{d y_{o}(1)}{d x}=0.0$

Then the strip function (4) becomes

$y_{o}(x)=x^{2}-2 x^{3}+x^{4}$

Equation (6) is the same as the strip function developed by Bhat [16]. The subsequent orthogonal polynomial functions are generated from Gram-Schmidt process [19]. The first orthogonal function $y_{1}(x)$ is obtained from $y_{1}(x)=\left(x-B_{1}\right) y_{o}(x)$

where $B_{1}=\underset{0}{\grave{\mathrm{o}}^{x}} x y_{o}^{2} d x / \underset{0}{\grave{\mathrm{o}}_{0}} y_{o}^{2} d x$. The higher terms in the set can be obtained by the recurrence relation between three successive members of the orthogonal polynomials set, i.e. $y_{k}(x)=\left(x-B_{k}\right) y_{k-1}-C_{k} y_{k-2}, k^{3} 2 \quad$ where
All the orthogonal polynomials that generated are satisfied the geometry and natural boundary conditions of the beam.

\section{PROBLEM FORMULATION:}

Assume a homogenous, isotropic, and linearly elastic rectangular plate in (x-y) plane as depicted in Fig. 1. The plate is of uniform in $\mathrm{x}$-direction and varying thickness $h(y)$ in y-direction. Under the assumption of the classical deformation theory, the partial differential equation governing the vibration of such plate is given by

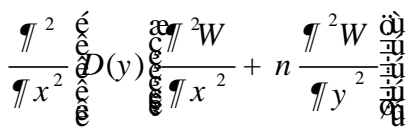

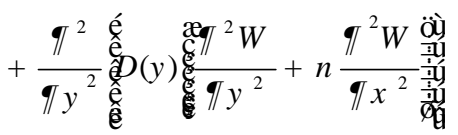

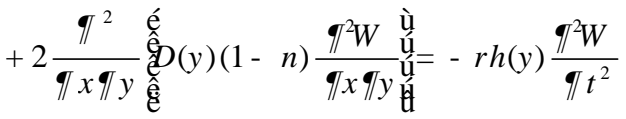

where $D(y)=\frac{E h^{3}(y)}{12\left(1-n^{2}\right)}$ is the flexure rigidity of the plate, $v$ is the Poisson's ratio, $E$ the Young's modulus, $r$ is the material density, and $W$ is the deflection of the plate. By normalizing the coordinate system with respect to the plate dimensions as follows: $x=\frac{x}{a}$ and $h=\frac{y}{b}$ and the variable thickness $h(y)$ with respect to the thickness of the plate $h_{o}$ at $h=0$ as $f(h)=\frac{h(h)}{h_{0}}$, equation (7) can be written as

$$
\begin{aligned}
& W_{x x x x}+2 b^{2} W_{x x h h}+b^{4} W_{h h h h}+2 b^{2} f_{1}(h)+\left\{b^{2} W_{h h h}+W_{x x h}\right\} \\
& +b^{2} f_{2}(h) \quad\left\{\begin{array}{lll}
b^{2} & W_{h h}+n & W_{x x}
\end{array}\right\}-l^{2} f_{3}(h) W=0,
\end{aligned}
$$

where $l^{2}=\frac{r h_{o} a^{4} w^{2}}{D_{0}}$ is the square of the natural frequency of the plate, $D_{o}$ is the flexural rigidity of the plate at $h=0, b=\frac{a}{b}$ is the aspect ratio of the plate and

$$
\begin{aligned}
& f_{1}(h)=\frac{1}{f^{3}(h)} \frac{d f^{3}(h)}{d h} \\
& f_{2}(h)=\frac{1}{f^{3}(h)} \frac{d^{2} f^{3}(h)}{d h^{2}} \\
& f_{3}(h)=\frac{1}{f^{2}(h)}
\end{aligned}
$$




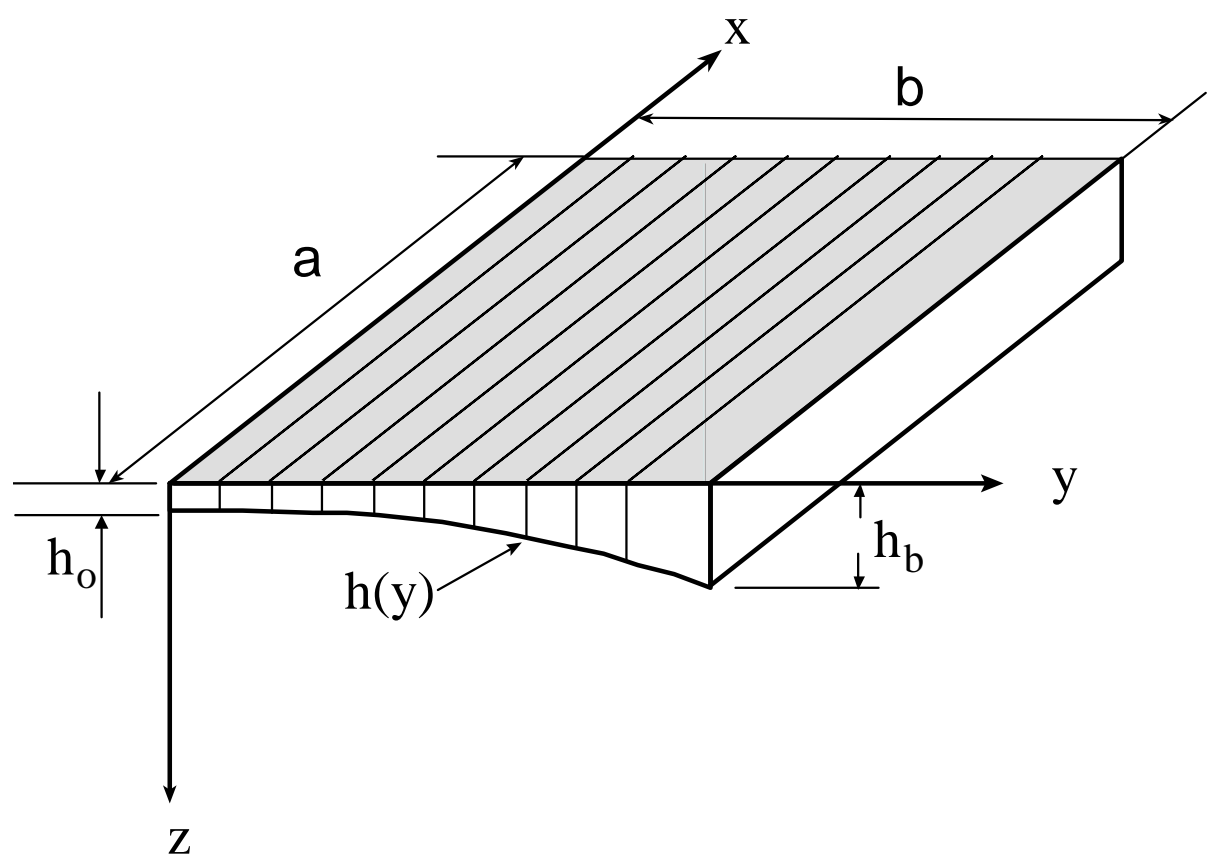

Fig. 1 Geometry of rectangular plate of variable thickness

For a plate striped in the $\mathrm{x}$-direction as shown in Fig.1, the shape function $W(x, h)$ may be assumed in the form

$W(x, h)=\stackrel{\circ}{\mathrm{a}}_{i=0}^{N} X_{i}(x) Y_{i}(h)$

where $Y_{i}(h)$ are the unknown functions in $h$-direction and $X_{i}(x)$ are the strip functions in $x$-direction that are developed in the previous section. Substituting equation (10), in equation (8), multiplying both sides by $X_{j}(x)$, and integrating from 0 to 1 , we may obtain

$$
\begin{gathered}
\stackrel{\stackrel{\mathrm{a}}{\mathrm{a}} q_{i j} Y_{i}}{ }+2 b^{2} n_{i j} Y_{i}^{\prime \prime}+b^{4} m_{i j} Y_{i}^{i v}+2 b^{2} f_{1}\left\{b^{2} m_{i j} Y_{i}^{\prime \prime \prime}+n_{i j} Y_{i}^{\prime}\right\} \\
+b^{2} f_{2}\left\{b^{2} m_{i j} Y_{i}^{\prime \prime}+n n_{i j} Y_{i}\right\}-l^{2} f_{3} m_{i j} Y_{i}=0 \\
\mathrm{j}=0,1,2, . ., \mathrm{N}
\end{gathered}
$$

where

$$
\begin{gathered}
m_{i j}=\underset{0}{\grave{\mathbf{o}}^{1}} X_{i}(x) X_{j}(x) d x, \\
n_{i j}=\grave{0}_{0}^{1} \frac{d^{2} X_{i}(x)}{d x^{2}} X_{j}(x) d x, \\
q_{i j}=\underset{0}{\grave{\mathbf{O}}} \frac{d^{4} X_{i}(x)}{d x^{4}} X_{j}(x) d x
\end{gathered}
$$

From the orthogonality properties, $m_{i j}=q_{i j}=0$ for $i^{1} j$, this is true for clamped-clamped boundary conditions in the $\mathrm{x}$-direction. Therefore, equation (11) becomes

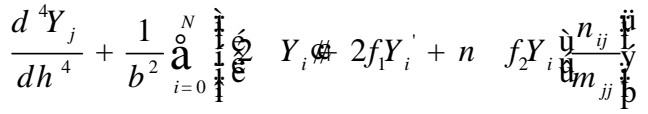

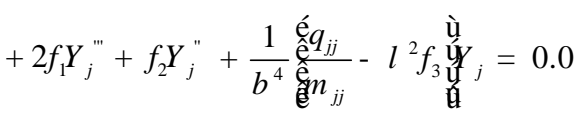

$$
\begin{aligned}
& \mathrm{j}=0,1,2, . . \mathrm{N}
\end{aligned}
$$

The above system of fourth order differential equations can be rewritten as 4-N first order differential equations at any strip $\mathrm{k}$ as:

$\frac{d \mathbf{Y}_{k}}{d h}=\{A\}_{k} \mathbf{Y}_{k}$

where the coefficients of the matrix $\{A\}_{k}$, in general, are functions of $h$ and the eigenvalue parameter $l$. The vector $\mathbf{Y}_{k}$ is given by

$$
\mathbf{Y}_{k}=\bar{G}_{\mathbb{E}_{1}} \bar{Y}_{2} . . \quad \bar{Y}_{i} . . \quad \bar{Y}_{N} \text { 狊 }
$$

where

$\overline{Y_{i}}=$ é $_{i} Y_{i} Y_{i}^{\prime \prime} Y_{i}{ }^{\prime \prime}$ ù

Solving the above system of first order differential equations using the transition matrix technique ${ }^{[20]}$ at any strip $k$, with boundaries $k-1$ and $k$ yield

$$
\mathbf{Y}_{k}=[\mathbf{B}] \quad \mathbf{Y}_{k-1}
$$


where $[\mathbf{B}]_{k}$ is called the transition matrix of the strip $k$ and $\mathbf{Y}_{k}, \mathbf{Y}_{k-1}$ are the nodal vectors of the boundaries $k, k-1$.

\section{BOUNDARY CONDITIONS:}

In this paper any combination of the classical boundary conditions such as simply supported, clamped, or free boundary conditions at either $\mathrm{y}=0$ or $\mathrm{y}=\mathrm{b}$ is considered. After normalizing the boundary conditions and using equation (10), we may have

$$
\frac{d^{2} Y_{j}}{d h^{2}}=0, Y_{j}=0 \quad(h=0, h=1),
$$

For simply supported edges,

$$
\frac{d Y_{j}}{d h}=0, Y_{j}=0 \quad(h=0, h=1) \text {. }
$$

For camped edges, and

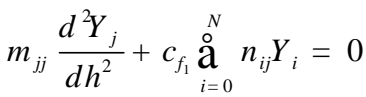

$$
\begin{aligned}
& m_{j j} \frac{d^{3} Y_{j}}{d h 3}+c_{f_{2}} \stackrel{\circ}{\mathrm{a}}_{i=0}^{N} n_{i j} \frac{d Y_{i}}{d h}=0 \quad(h=0, h=1)
\end{aligned}
$$

where $c_{f_{1}}=\frac{n}{b^{2}}$ and $c_{f_{2}}=\frac{(2-n)}{b^{2}}$ for free edges.

\section{NUMERICAL RESULTS AND CONCLUSION:}

To illustrate the validity of the proposed set of orthogonal polynomial strip functions in finite strip method, the natural frequencies for the free vibration plate of variable thickness in y-direction are calculated and compared with the available results obtained by different numerical methods. Numerical results are obtained for different combinations of classical boundary conditions, aspect ratio and tapered ratio.

A linearly tapered plate is assumed with nondimensional variable thickness function defined by

$f(h)=(1+d h)$

where $d$ is the taper ratio given by $d=\frac{h_{b}-h_{0}}{h_{0}}$, where $h_{b}$ is the thickness at $h=1$.

To investigate the accuracy and the convergence of the proposed admissible functions, the first four natural frequencies are calculated for different two cases, CSCS with taper ratio $d=0.5$, and CCCC with taper ratio $d=0.4$ as shown in table 1 . Different terms of the series solution in equation $(10)(N)$ are considered. Some of the results that are obtained by using classical beam functions are calculated and presented to compare the convergent between the two admissible functions. Also, the results compared with that obtained from Grossi and Bhat ${ }^{[9]}$ and Singh and Saxena ${ }^{[11]}$. For the purpose of brevity, the symbolism CFCS for example, means a plate with varying thickness in the y-direction having clamped, free, clamped and simple supported edges at the boundaries, $\mathrm{x}=0, \mathrm{y}=\mathrm{b}, \mathrm{x}=\mathrm{a}$, and $\mathrm{y}=0$, respectively (see Fig. 1). In all calculations, a Poisson's ratio $v=0.3$ was used.

Table 2 demonstrates the natural frequencies for several combinations of boundary conditions, for two taper ratiod $(0,0.5)$, and two aspect ratio $b \quad(1,2)$. A comparison is made with some results obtained from other numerical methods. Good accuracy has been seen for all the cases considered in this paper.

Table 1: Convergence of the first four frequencies of square plate of variable thickness and comparison with data from different techniques (CSCS and CCCC plates)

\begin{tabular}{ccccccc}
\hline & $\mathrm{N}$ & $\beta$ & $\delta$ & $\lambda_{1}$ & $\lambda_{2}$ & $\lambda_{3}$ \\
\hline Classic & 1 & 1 & .5 & 36.0213 & 68.3958 & 85.2728 \\
CSCS & 2 & & & 36.0213 & 68.3958 & 85.2728 \\
& 3 & & & 35.9716 & 68.0789 & 85.2411 \\
Polynomial & 4 & & & 35.9716 & 68.0789 & 86.2478 \\
CSCS & 1 & & & 35.9672 & 68.1654 & 86.2478 \\
& 3 & & & 35.9227 & 67.8702 & 86.2303 \\
Singh and Saxena & 5 & & & 35.9173 & 67.8270 & 86.2229 \\
Polynomial & 8 & & & 35.9165 & 67.8188 & 85.2000 \\
CCCC & & & & 35.9640 & 68.033 & 87.2030 \\
Singh and Saxena & 1 & 1 & .4 & 42.9255 & 87.6976 & 87.1805 \\
\end{tabular}


Table 2: The First four frequencies $\lambda_{\mathrm{i}}(\mathrm{i}=1,2,3,4)$ of tapered plate in $\mathrm{y}$-direction for different boundary conditions

\begin{tabular}{|c|c|c|c|c|c|c|c|}
\hline $\mathrm{N}$ & & $\beta$ & $\delta$ & $\lambda_{1}$ & $\lambda_{2}$ & $\lambda_{3}$ & $\lambda_{4}$ \\
\hline 5 & CCCS & 1 & 0.0 & 31.7809 & 63.1909 & 71.8811 & 100.879 \\
\hline 5 & & & 0.5 & 39.6479 & 89.7691 & 77.9645 & \\
\hline 5 & & 2 & 0.0 & 73.2638 & 108.272 & 168.863 & 210.392 \\
\hline \multirow[t]{2}{*}{5} & & & 0.5 & 88.0933 & 134.256 & 211.415 & \\
\hline & Singh & $n a^{[11]}$ & & 88.2750 & 134.190 & 206.890 & \\
\hline \multirow[t]{2}{*}{5} & $\mathrm{CSCC}$ & 1 & 0.5 & 39.2255 & 78.7977 & 87.3291 & \\
\hline & Singh & $\mathrm{na}^{[11]}$ & & 39.2820 & 78.9690 & 86.404 & \\
\hline \multirow[t]{2}{*}{5} & & 2 & 0.5 & 93.6774 & 134.575 & 206.9541 & 93.6773 \\
\hline & Singh & $\mathrm{na}^{[11]}$ & & 93.8310 & 134.520 & 202.580 & \\
\hline 5 & CFCC & 1 & 0.0 & 24.2741 & 40.2840 & 62.9298 & \\
\hline 5 & & & 0.5 & 27.6150 & 49.8425 & 68.0844 & 95.9289 \\
\hline 5 & & 2 & 0.0 & 31.8625 & 63.3429 & 103.4920 & 127.067 \\
\hline 5 & & & 0.5 & 39.5637 & 75.8020 & 133.582 & 144.134 \\
\hline 5 & CFCF & 1 & 0.0 & 22.3639 & 26.7401 & 43.8835 & 62.9323 \\
\hline 5 & & & 0.5 & 26.8121 & 34.3755 & 54.7061 & 68.0833 \\
\hline 5 & & 2 & 0.0 & 22.3197 & 36.5568 & 62.9065 & 62.9389 \\
\hline 5 & CFCS & 1 & 0.5 & 27.1122 & 44.2948 & 68.0726 & 91.3042 \\
\hline 5 & & 2 & 0.0 & 26.9892 & 62.9285 & 80.0701 & 108.2721 \\
\hline 5 & & & 0.5 & 32.0426 & 74.2904 & 101.6199 & 142.2618 \\
\hline 5 & CSCS & 1 & 0.0 & 28.9125 & 54.5913 & 70.1724 & 94.7093 \\
\hline 5 & & & 0.5 & 35.9173 & 67.8270 & 86.2303 & 126.5393 \\
\hline 5 & & 2 & 0.0 & 54.5901 & 94.7081 & 158.9818 & 170.1827 \\
\hline 5 & & & 0.5 & 67.7910 & 117.7756 & 196.9454 & 211.1377 \\
\hline
\end{tabular}

\section{CONCLUSION REMARKS:}

The finite strip transition matrix method (FSTM) by using new set of orthogonal polynomial strip functions presented in this paper is used to investigate the flexure vibration of plate of generally variable thickness with general classical boundary conditions. The method is easily implemented in a computer program and yield a fast convergence and reliable results. The results obtained by the proposed method are compared with known results from different numerical and approximate methods. Excellent agreement has been achieved for all plate configurations considered in this paper. The comparison results illustrate the high accuracy and efficiency of the present method. Other complexity effects such as orthotropic, edges restrained against translation and rotation, concentrated masses, etc., can be easily implemented in the present method.

\section{REFERENCES:}

[1] A. W. Leissa, "Vibration of plates (NASA sp 160)", Washington, D.C., U.S. Governments Printing Office, 1969.

[2] A. W. Leissa, "The Free Vibration of Rectangular Plates", Journal of Sound and Vibration 31, pp. 257-293, 1973.

[3] A. W. Leissa, " Recent Research in Plate Vibration. 1973- 1976Classical Theory", The Shock and Vibration Digest.10., (1977), pp.13-24, 1977.
[4] A. W. Leissa,,"Plate Vibration Research. 1976-1980: Classical Theory", The Shock and Vibration Digest.13, pp. 11-22, 1981.

[5] A. W. Leissa,.," Recent Studies in Plate Vibrations. 1981-1985, Part I, Classical Theory", The Shock and Vibration Digest.19, pp. 11-18, 1987,.

[6] A. S. Ashour, "A Semi-Analytical Solution of The Flexural Vibration of Orthotropic Plates of Variable Thickness", Journal of Sound and Vibration, in press, 2000.

[7] Y. K. Cheung and D. Zhou, " The free vibration of Tapered Rectangular Plates Using New Set of Beam Functions with The Rayleigh-Ritz Method", Journal of Sound and Vibration223, pp. 703-722, 1999.

[8] D. Zhou, " Natural Frequencies of Rectangular Plates Using a Set Of Static Beam Function in Rayleigh-Ritz Method" , Journal of Sound and Vibration 189, pp. 8187, 1996.

[9] R. O. Gross and R.B. Bhat," Natural Frequencies of Edge Restrained Tapered Rectangular Plates" , Journal of Sound and Vibration 185, pp. 335-343, 1995.

[10] B. Singh and V. Saxena, " Transverse Vibration of Skew Plates with Variable Thickness", Journal of Sound and Vibration 206, pp. 1-13, 1997.

[11] B. Singh. and V. Saxena, " Transverse Vibration of Rectangular Plate with Bi-directional Thickness Variation", Journal of Sound and Vibration 198, pp. 5165, 1996.

[12] B. Singh and V. Saxena, " Flexural Vibration of Skew Plate Using Boundary Characteristic Orthogonal Polynomials in Two Variables", Journal of Sound and Vibration 173, pp. 157- 178, 1994. 
[13] K. M. Liew and K. L. Lam," Application of Two Dimensional Orthogonal Plate Function to Flexural Vibration of Skew Plates", Journal of Sound and Vibration 139, pp. 241-252, 1990.

[14] R. B. Bhat," Flexura Vibration of Polygonal Plates Using Characteristic Orthogonal Polynomials in Two Variables", Journal of Sound and Vibration 114, pp. 65$71,1987$.

[15] S. K. Malhotra, N. Ganesan and M.A. Veluswami, "Vibration of Orthotropic Square Plate Having Variable Thickness (Parabolic Shape)", Journal of Sound and Vibration 119, pp. 184-188, 1987.

[16] R.B. Bhat, "Natural Frequencies of Rectangular Plates Using Characteristic Orthogonal Polynomials in Rayleigh-Ritz Method", Journal of Sound and Vibration 102, pp. 493-499, 1985.

[17] A. M. Farag, "Mathematical Analysis of Free and Forced Vibration of Rectangular Plate", Ph.D. thesis, Faculty of Engineering , Alexandria University, Alexandria, Egypt, 1994.

[18] A.M. Farag and A.S. Ashour, "Free Vibration of Orthotropic Skew Plates" , Journal of Vibration and Acoustics Vol. 122, pp. 313-317, 2000.

[19] S.J. Leon, Linear Algebra with Applications. New Jersey, Prentic-Hall International, Inc. Saddle River, 1998.

[20] R. Zurmuhl, Numerical Analysis for Engineers and Physicists. , New York, Springer-Verlag Berlin Heidelberg, 1976.

\section{BIOGRAPHIES}

Dr. Abd El- Fattah A. Rizk is currently Associate Professor at the faculty of Engineering, International Islamic University Malaysia, Malaysia, on leave from Faculty of Engineering, Alexandria University, Alexandria, Egypt. He was born in Alexandria 1952. He obtained his B.Sc. in Mechanical Engineering in 1975, Faculty of Engineering, Alexandria University, Egypt, and B.Sc. in Mathematics in 1979, Faculty of Science, Alexandria University, Egypt. He received his M.Sc. in 1984 and Ph.D. in 1988 from Faculty of Engineering, Lehigh University, USA. His area of interest is Solid and Fracture Mechanics.

Ahmed A. S. Ashour was born in Alexandria, Egypt in 1959. He received his bachelor's degree in 1982 in Telecommunications Engineering, his MSc. in dynamical system model for traffic noise in 1988 from Alexandria University. He obtained his MSc in Engineering Mechanics in 1992 and his $\mathrm{PhD}$ in Acoustics in 1994 from The University of Texas at Austin, which was comprised of a study of the fracture impedance method. His research interests include wave propagation in solids and fluid, structural vibration and noise, and mathematical modeling and simulation. $\mathrm{He}$ is an Associate Professor at Alexandria University, Egypt. Currently, He is Associate Professor and head, Department of Science in Engineering, International Islamic University Malaysia. 